Pacific Journal of Mathematic 


\title{
METRIZABILITY OF TOPOLOGICAL SPACES
}

\author{
R. E. HODEL
}

This paper is a study of conditions under which a topological space is metrizable or has a countable base. In $\S 2$ we consider the metrizability of spaces having a weak base in the sense of Arhangel'skir. In $\S 3$ we extend earlier work of Bennett on quasi-developments by showing that every regular $\theta$-refinable $\beta$-space with a quasi- $G_{\delta}$-diagonal is semi-stratifiable. One consequence of this result is a generalization of the Borges-Okuyama theorem on the metrizability of a paracompact $w \Delta$-space with a $G_{\delta}$-diagonal. In $\S 4$ we prove that a regular space has a countable base if it is hereditarily a $C C C w \Delta$-space with a point-countable separating open cover. This result is motivated by the remarkable theorem of Arhangel'skii which states that a regular space has a countable base if it is hereditarily a Lindelöf $p$-space. In $\S 5$ we show that every regular $p$-space which a Baire space has a dense subset which is a paracompact $p$-space. This result, related to work of Sapirovskir, is then used to obtain conditions under which a Baire space satisfying the $C C C$ is separable or has a countable base. In $\S 6$ we prove that every locally connected, locally peripherally separable meta-Lindelöf Moore space is metrizable. Finally, in $\S 7$ we consider the metrizability of spaces which are the union of countably many metrizable subsets. The results obtained in this section extend earlier work of Čban, Corson-Michael, Smirnov, and Stone.

1. Preliminaries. We begin with some definitions and known results which are used throughout this paper. Unless otherwise stated, no separation axioms are assumed; however, regular, normal, and collectionwise normal spaces are always $T_{1}$ and paracompact spaces are always Hausdorff. The set of natural numbers is denoted by $\boldsymbol{N}$, and $i, j, k, m, n, r, s$, and $t$ denote elements of $\boldsymbol{N}$.

Let $X$ be a set, let $\mathscr{G}$ be a collection of subsets of $X$, let $p$ be an element of $X$. The star of $p$ with respect to $\mathscr{G}$, denoted $\operatorname{st}(p, \mathscr{G})$, is the union of all elements of $\mathscr{G}$ containing $p$. The order of $p$ with respect to $\mathscr{G}$, denoted ord $(p, \mathscr{G})$, is the number of elements of $\mathscr{G}$ containing $p$. The union of all elements of $\mathscr{G}$ is denoted by $\mathscr{G}^{*}$. If $\mathscr{G}$ covers $X$, then $\mathscr{G}$ is said to be separating [43] if given any two distinct points $p$ and $q$ in $X$, there is some $G$ in $\mathscr{G}$ such that $p \in G$, $q \notin G$.

A topological space $X$ is said to be developable if there is a sequence $\mathscr{G}_{1}, \mathscr{G}_{2}, \cdots$ of open covers of $X$ such that, for each $p$ in $X$, $\left\{\operatorname{st}\left(p, \mathscr{G}_{n}\right): n=1,2, \cdots\right\}$ is a fundamental system of neighborhoods of 
$p$. Such a sequence of open covers is called a development for $X$. A regular developable space is called a Moore space. Bing [12] proved that every paracompact Moore space is metrizable.

A quasi-development for a topological space $X$ is a sequence $\mathscr{G}_{1}$, $\mathscr{G}_{2}, \cdots$ of open collections in $X$ such that, given any point $p$ in $X$ and any neighborhood $R$ of $p$, there is some $n$ in $N$ such that st $\left(p, \mathscr{G}_{n}\right) \neq \phi$ and st $\left(p, \mathscr{G}_{n}\right) \subseteq R$. Clearly every development is a quasidevelopment, and in [10] Bennett proved that a space is developable if it has a quasi-development and every closed subset is a $G_{\dot{j}}$.

The notion of a $G_{\dot{o}}$-diagonal plays an important role in metrization theory; see, for example, [13], [14], [18], [51]. In [18] Ceder proved that a space $X$ has a $G_{i}$-diagonal if and only if there is a sequence $\mathscr{G}_{1}, \mathscr{G}_{2}, \cdots$ of open covers of $X$ such that, given any two distinct points $p$ and $q$ in $X$, there is some $n$ in $N$ such that $q \in \operatorname{st}\left(p, \mathscr{G}_{n}\right)$. Such a sequence of open covers is called a $G_{\hat{o}}$-diagonal sequence for $X$.

A collection $\mathscr{G}$ of subsets of a topological space $X$ is called a net [6] if given any point $p$ in $X$ and any neighborhood $R$ of $p$, there is some $G$ in $\mathscr{G}$ such that $p \in G \subseteq R$. A space with a $\sigma$-locally finite net is called a $\sigma$-space [50].

A topological space $X$ is $\theta$-refinable [64] if for each open cover $\mathscr{Q}$ of $X$, there is a sequence $\mathscr{G}_{1}, \mathscr{G}_{2}, \cdots$ of open refinements such that, for each $p$ in $X$, there is some $n$ in $N$ such that $\operatorname{ord}\left(p, \mathscr{G}_{n}\right)$ is finite. Such a sequence of open covers is called a $\theta$-refinement of $\mathscr{Q}$.

A topological space $X$ is a $w \Delta$-space [14] if there is a sequence $\mathscr{G}_{1}, \mathscr{G}_{2}, \cdots$ of open covers of $X$ such that, for each $p$ in $X$, if $x_{n} \in$ st $\left(p, \mathscr{G}_{n}\right)$ for $n=1,2, \cdots$, then the sequence $\left\langle x_{n}\right\rangle$ has a cluster point. Clearly every countably compact space and every developable space is a $w \Delta$-space. In [31] it is proved that every regular $w \Delta$-space with a $\sigma$-point finite separating open cover is developable.

The $p$-spaces of Arhangel'skii [4], which generalize Cech complete spaces and completely regular developable spaces, are defined in terms of the Stone-Cech compactification. In this paper we adopt as the definition of a $p$-space an internal characterization given by Burke in [16]. A pluming for a topological space $X$ is a sequence $\mathscr{G}_{1}, \mathscr{G}_{2}, \cdots$ of open covers of $X$ such that, for each $p$ in $X$, if $p \in G_{n} \in \mathscr{G}_{n}, n=$ $1,2, \cdots$, then (a) $C(p)=\bigcap_{n=1}^{\infty} \bar{G}_{n}$ is compact; (b) $\left\{\bigcap_{i=1}^{n} \bar{G}_{i}: n=1,2, \cdots\right\}$ is a "base" for $C(p)$ in the sense that given any open set $R$ with $C(p) \subseteq R$, there is some $n$ in $N$ such that $\bigcap_{i=1}^{n} \bar{G}_{\imath} \subseteq R$. A topological space is a $p$-space if it has a pluming.

Quasi-complete spaces were introduced by Creede in [22] as a generalization of $p$-spaces and $w \Delta$-spaces. In this paper we adopt as the definition of quasi-completeness a characterization due independently to Sohn-Choi [61] and Gittings [25]. A topological space $X$ is quasi- 
complete if there is a sequence $\mathscr{G}_{1}, \mathscr{G}_{2}, \cdots$ of open covers of $X$ such that the following holds for each $p$ in $X$ : if $p \in G_{n} \in \mathscr{G}_{n}, n=1,2, \cdots$, and $\left\langle x_{n}\right\rangle$ is a sequence with $x_{n} \in \bigcap_{i=1}^{n} G_{i}$ for all $n$, then $\left\langle x_{n}\right\rangle$ has a cluster point. Such a sequence of open covers is called a quasi-complete sequence for $X$. In [16] Burke proved that $w \Delta$-spaces and $p$-spaces coincide for the class of completely regular $\theta$-refinable spaces. Recently Gittings [25] extended this result by showing that $p$-spaces (as defined above), $w \Delta$-spaces, and quasi-complete spaces coincide for the class of regular $\theta$-refinable spaces.

Recall that a topological space is called a Baire space if any countable intersection of open dense sets is dense, and is $\boldsymbol{\aleph}_{1}$-compact if every uncountable subset has a limit point. Also, a topological space is a CCC space, or satisfies the CCC (CCC = countable chain condition), if every mutually disjoint collection of nonempty open sets is countable. It is easy to check that a topological space is hereditarily $\aleph_{1}$-compact if and only if it hereditarily satisfies the CCC.

Let $(X, \mathscr{T})$ be a topological space, let $g$ be a function from $N \times X$ into $\mathscr{T}$ such that $p \in \bigcap_{n=1}^{\infty} g(n, p)$ for each $p$ in $X$. Consider the following conditions on $g$.

(A) If $g(n, p) \cap g\left(n, x_{n}\right) \neq \phi$ for all $n$, then $p$ is a cluster point of $\left\langle x_{n}\right\rangle$.

(B) If $g(n, p) \cap g\left(n, x_{n}\right) \neq \phi$ for all $n$, then $\left\langle x_{n}\right\rangle$ has a cluster point.

(C) If $y_{n} \in g(n, p)$ and $x_{n} \in g\left(n, y_{n}\right)$ for all $n$, then $p$ is a cluster point of $\left\langle x_{n}\right\rangle$.

(D) If $x_{n} \in g(n, p)$ for all $n$, then $\left\langle x_{n}\right\rangle$ has a cluster point.

(E) If $p \in g\left(n, x_{n}\right)$ for all $n$, then $\left\langle x_{n}\right\rangle$ has a cluster point.

In [29] Heath characterized Nagata spaces in terms of a function $g$ satisfying (A). (The $T_{0}$ separation axiom is assumed.) A function $g$ satisfying (A) is called a Nagata function. A topological space $X$ is, respectively, a $w N$-space, a $\gamma$-space, a $q$-space, a $\beta$-space if it has a function $g$ satisfying, respectively, (B), (C), (D), (E). A function $g$ satisfying $(\mathrm{C})$ is called a $\gamma$-function, and one satisfying (E) is called

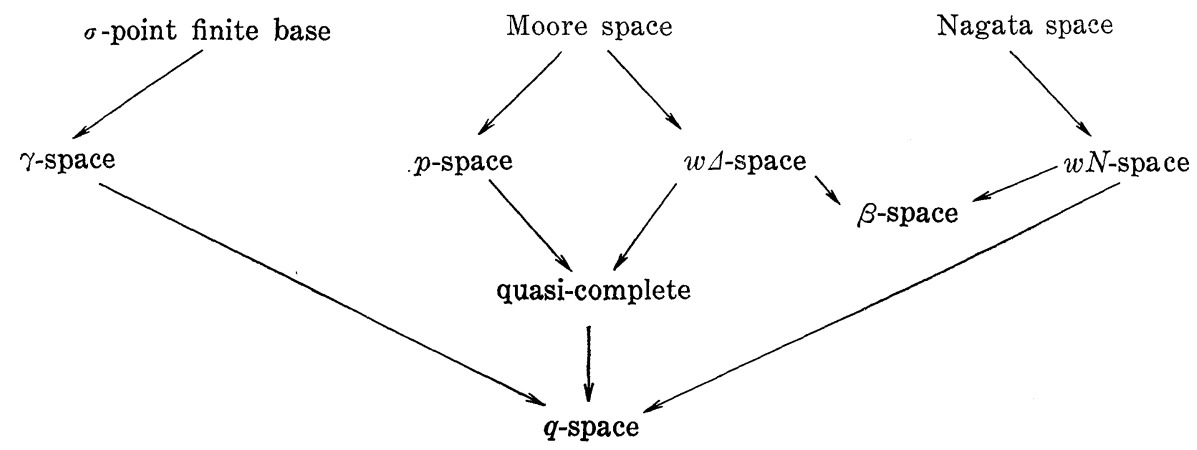


a $\beta$-function. See [31], [32], [41]. The idea of characterizing generalized metrizable spaces in the above fashion was first used by Heath in [30].

The relationship between some of the classes of spaces defined in this section is summarized in the preceding diagram.

2. Weak bases. The following natural technique for defining a topology on a set is due to Arhangel'skii [5]. (Also, see [56].) Let $X$ be a set, and suppose that for each $p$ in $X$ there is assigned a collection $\mathscr{B}_{p}$ of subsets of $X$ which is closed under finite intersections such that each element of $\mathscr{B}_{p}$ contains $p$. Let $\mathscr{T}$ be all subsets $V$ of $X$ satisfying this condition: for each $p$ in $V$, there is some $B_{p}$ in $\mathscr{B}_{p}$ such that $B_{p} \subseteq V$. As is easily seen, the collection $\mathscr{T}$ obtained in this fashion is a topology on $X$. This construction suggests the following definitions.

Let $X$ be a topological space, and for each $p$ in $X$ let $\mathscr{B}_{p}$ be a collection of subsets of $X$ which is closed under finite intersections such that each element of $\mathscr{B}_{p}$ contains $p$. The collection $\mathscr{B}=\left\{\mathscr{B}_{p}\right.$ : $p$ in $X\}$ is called a weak base for $X$ [5] if the following condition holds: a subset $V$ of $X$ is open if and only if for each $p$ in $V$, there is some $B_{p}$ in $\mathscr{B}_{p}$ such that $B_{p} \subseteq V$. Now let $\mathscr{G}_{1}, \mathscr{G}_{2}, \cdots$ be a sequence of (not necessarily open) covers of a topological space $X$ such that $\mathscr{G}_{n+1}$ refines $\mathscr{G}_{n}, n=1,2, \cdots$. Such a sequence of covers is said to be semi-refined [19] if $\left\{\mathscr{B}_{p}: p\right.$ in $\left.X\right\}$ is a weak base for $X$, where $\mathscr{B}_{p}=\left\{\right.$ st $\left.\left(p, \mathscr{G}_{n}\right): n=1,2, \cdots\right\}$. The two theorems in this section give conditions under which a weak base and a semi-refined sequence of covers yield metrizability. The first result is closely related to the general metrization theorem of Nagata [48], while the second result generalizes the metrization theorem of Alexandroff and Urysohn [1].

THEOREM 2.1. A $T_{0}$ space $(X, \mathscr{T})$ is metrizable if and only if for each $p$ in $X$ there is a collection $\mathscr{B}_{p}=\{B(n, p): n=1,2, \cdots\}$ of subsets of $X$ satisfying these conditions:

(1) $p \in B(n, p)$ and $B(n+1, p) \subseteq B(n, p)$;

(2) $q \in B(n+1, p)$ implies $B(n+1, q) \leqq B(n, p)$;

(3) $q \notin B(n, p)$ implies $B(n+1, p) \cap B(n+1, q)=\phi$;

(4) $\left\{\mathscr{B}_{p}: p\right.$ in $\left.X\right\}$ is a weak base for $X$.

Proof. If $X$ is metrizable, the existence of the collections is clear. Assume, then, that for each $p$ in $X$ there is a collection $\mathscr{B}_{p}=$ $\{B(n, p): n=1,2, \cdots\}$ of subsets of $X$ satisfying (1)-(4). To prove $X$ metrizable, it suffices to show that there is a function $g: N \times X \rightarrow \mathscr{T}$ (with $p \in \bigcap_{n=1}^{\infty} g(n, p)$ for all $p$ ) which is a $\gamma$-function and a Nagata function. (See [32].) For $p$ in $X, n$ in $N$ let $g(n, p)=B(n, p)^{0}$. First 
let us show that $p \in g(n, p)$. Let

$$
V=\left\{x \text { in } X: B\left(j_{x}, x\right) \subseteq B(n, p) \text { for some positive integer } j_{x}\right\} \text {. }
$$

It is clear that $p \in V \subseteq B(n, p)$, and so it remains to show that $V$ is open. Suppose it is not. Then by (4), there is some $q$ in $V$ such that $B(k, q) \nsubseteq V, k=1,2, \cdots$. Now $q \in V$, so $B(j, q) \subseteq B(n, p)$ for some $j$. But $B(j+1, q) \nsubseteq V$, so there exists $x \in B(j+1, q)$ such that $x \notin V$. Since $x \notin V, B(j+1, x) \nsubseteq B(n, p)$, so there exists $y \in B(j+1, x)$ such that $y \notin B(n, p)$. Now $x \in B(j+1, q)$, so by $(2), B(j+1, x) \subseteq$ $B(j, q)$. Hence $y \in B(n, p)$, a contradiction. Thus $V$ is an open set.

To see that $g$ is a Nagata function, let $g(n, p) \cap g\left(n, x_{n}\right) \neq \phi, n=$ $1,2, \cdots$, and let us show that $x_{n} \rightarrow p$. Let $V$ be an open neighborhood of $p$. By (4), $B\left(n_{0}, p\right) \subseteq V$ for some $n_{0}$. Then for $n \geqq n_{0}+1$, $x_{n} \in V$. Indeed, since $B(n, p) \cap B\left(n, x_{n}\right) \neq \phi$, it follows from (3) that $x_{n} \in B(n-1, p)$, and hence $x_{n} \in V$.

To see that $g$ is a $\gamma$-function, let $y_{n} \in g(n, p), x_{n} \in g\left(n, y_{n}\right)$, and let us show that $x_{n} \rightarrow p$. Let $V$ be an open neighborhood of $p$. By (4), $B\left(n_{0}, p\right) \subseteq V$ for some $n_{0}$. Then for $n \geqq n_{0}+1, x_{n} \in V$. Indeed, since $y_{n} \in B(n, p)$, it follows from (2) that $B\left(n, y_{n}\right) \subseteq B(n-1, p)$. Since $x_{n} \in B\left(n, y_{n}\right)$ and $B(n-1, p) \subseteq B\left(n_{0}, p\right) \subseteq V$, it follows that $x_{n} \in V$.

THeOREM 2.2. A $T_{0}$ space $X$ is metrizable if and only if there is a sequence $\mathscr{G}_{1}, \mathscr{G}_{2}, \ldots$ of covers of $X$ satisfying these conditions.

(1) The sequence $\mathscr{G}_{1}, \mathscr{G}_{2}, \cdots$ is semi-refined;

(2) for all $n$, if $G_{1}$ and $G_{2}$ are elements of $\mathscr{G}_{n+1}$ such that $G_{1} \cap G_{2} \neq \phi$, then $G_{1} \cup G_{2} \leqq G$ for some $G$ in $\mathscr{G}_{n}$.

Proof. If $X$ is metrizable, the existence of the covers is clear. Assume, then, that $\mathscr{G}_{1}, \mathscr{G}_{2}, \cdots$ is a sequence of covers satisfying (1) and (2). For $p$ in $X, n$ in $N$ let $B(n, p)=\operatorname{st}\left(p, \mathscr{G}_{n}\right)$, and let $\mathscr{B}_{p}=$ $\{B(n, p): n=1,2, \cdots\}$. It is easy to check that the collections $\left\{\mathscr{B}_{p}\right.$ : $p$ in $X$ \} satisfy the conditions of Theorem 2.1, and hence $X$ is metrizable.

3. Spaces with a quasi- $G_{\delta}$-diagonal. A paracompact $w \Delta$-space is metrizable if it has any one of the following: (1) a $G_{\tilde{o}}$-diagonal (Borges [13], Okuyama [51]); (2) a point-countable base (Filippov [24]); (3) a point-countable separating open cover (Nagata [47]); (4) a quasidevelopment (Bennett [9]). The notion of a point-countable separating open cover was introduced by Nagata to achieve a unification of (1) and (2). The problem of obtaining a similar unification of (1) and (4) suggests the following property. A quasi-G $G_{i}$-diagonal for a topological space $X$ is a sequence $\mathscr{G}_{1}, \mathscr{G}_{2}, \ldots$ of open collections in $X$ such that, 
given any two distinct points $p$ and $q$ in $X$, there is some $n$ such that st $\left(p, \mathscr{G}_{n}\right) \neq \phi$ and $q \notin \operatorname{st}\left(p, \mathscr{G}_{n}\right)$.

Recently Bennett and Berney [11] proved that every regular $\theta$ refinable $\beta$-space with a quasi-development is developable. This result yields the following generalization of (4): every paracompact $\beta$-space with a quasi-development is metrizable.

In this section we modify Bennett's proof of (4) to obtain the result that every regular $\theta$-refinable $\beta$-space with a quasi- $G_{\tilde{o}}$-diagonal is semi-stratifiable. Consequences of this result include a unification of (1) and (4) and the above result of Bennett and Berney on developable spaces. As in Bennett's proof of (4), use is made of the following set-theoretical lemma. (See Theorem 114 on p. 47 of [45].)

Lemma 3.1. Let $Q$ be a property, let $\mathscr{H}_{1}, \mathscr{H}_{2}, \cdots$ be finite nonempty sets, and assume that for all $n$, each element of $\mathscr{H}_{n+1}$ has property $Q$ with respect to some element of $\mathscr{H}_{n}$. Then there is a sequence $H_{1}, H_{2}, \cdots$ such that for all $n, H_{n} \in \mathscr{H}_{n}$ and $H_{n+1}$ has property $Q$ with respect to $H_{n}$.

THEOREM 3.2. Every regular $\theta$-refinable $\beta$-space with a quasi$G_{\tilde{\delta}}$-diagonal is semi-stratifiable.

Proof. Let $(X, \mathscr{T})$ be a regular $\theta$-refinable space, let $l: N \times X \rightarrow$ $\mathscr{T}$ be a $\beta$-function for $X$ (assume that $l(n+1, p) \leqq l(n, p), n=1,2$, $\cdots$, and all $p$ in $X)$, and let $\mathscr{G}_{1}, \mathscr{G}_{2}, \cdots$ be a quasi- $G_{\dot{\delta}}$-diagonal for $X$. Assume, for a moment, that every closed subset of $X$ is a $G_{\hat{o}}$. It easily follows that $X$ has a $G_{i}$-diagonal. Indeed, for $n=1,2, \cdots$ let $\mathscr{G}_{n}^{*}=\bigcup_{k=1}^{\infty} F_{n k}$, where each $F_{n k}$ is a closed set, and let $\mathscr{H}_{n k}=$ $\mathscr{G}_{n} \cup\left\{X-F_{n k}\right\}$. Then, as is easy to check, $\left\{\mathscr{H}_{n k}: n, k\right.$ in $\left.N\right\}$ is a $G_{\delta^{-}}$ diagonal sequence for $X$. Since every regular $\theta$-refinable $\beta$-space with a $G_{\delta}$-diagonal is semi-stratifiable (see [31]), the proof is complete.

It remains to show that every closed set is a $G_{\delta}$. So let $M$ be a closed subset of $X$, and assume that $M$ has no isolated points. (Let $I$ be the isolated points of $M$. If $M-I$ is a $G_{\delta}$, it easily follows that $M$ itself is a $G_{\dot{o}}$.) For each $x$ in $M$, there is a strictly increasing sequence of positive integers $m(1, x)<m(2, x)<\cdots<m(i, x)<\cdots$ such that for all $n, x \in \mathscr{G}_{n}^{*}$ if and only if $n=m(i, x)$ for some $i \leqq n$. For $i=1,2, \cdots$ let $G(i, x)$ be some element of $\mathscr{G}_{m(i, x)}$ containing $x$.

For each $x$ in $M$, let $H(1, x)=l(1, x) \cap G(1, x)$. Then $\{H(1, x)$ : $x \in M\}$, together with $(X-M)$, covers $X$. Let $\left\{\mathscr{W}_{1, k}: k=1,2, \cdots\right\}$ be a $\theta$-refinement of this cover. Note that if $W$ is any element of $\mathscr{W}_{1, k}$ such that $W \cap M \neq \phi$, then there is some $x$ in $M$ such that $W \cong$ $H(1, x)$.

Next, for each $x$ in $M$, let $V(2, x)$ be an open neighborhood of $x$ 
such that $\overline{V(2, x)}$ is contained in some element of $\mathscr{W}_{1,1}$. Let $H(2, x)=$ $V(2, x) \cap l(2, x) \cap G(1, x) \cap G(2, x)$. Then $\{H(2, x): x$ in $M\}$, together with $(X-M)$, covers $X$. Let $\left\{\mathscr{W}_{2, k}: k=1,2, \cdots\right\}$ be a $\theta$-refinement of this cover.

Continue this process. Then for each $n$ we obtain a sequence $\mathscr{W}_{n, 1}, \mathscr{W}_{n, 2}, \cdots$ of open covers of $X$, and a collection $\{H(n, x): x \in M\}$ of open sets, such that the following hold for all $n$ : in $M$;

(1) if $W \in \mathscr{W}_{n, k}$ and $W \cap M \neq \phi$, then $W \subseteq H(n, x)$ for some $x$

(2) for all $x$ in $M, x \in H(n, x) \subseteq l(n, x) \cap\left(\bigcap_{i=1}^{n} G(i, x)\right)$;

(3) for all $x$ in $M$ and all $j \leqq n, k \leqq n, \overline{H(n+1, x)}$ is contained in some element of $\mathscr{W}_{j, k}$;

(4) for all $x$ in $X$, there is some $k$ such that ord $\left(x, \mathscr{W}_{n, k}\right)$ is finite.

Now let $V_{n, k}=\cup\left\{W \in \mathscr{W}_{n, k}: W \cap M \neq \phi\right\}$. Clearly each $V_{n, k}$ is open and $M \subseteq \bigcap_{n, k=1}^{\infty} V_{n, k}$. Let $p \in \bigcap_{n, k=1}^{\infty} V_{n, k}$, but suppose $p \notin M$. By (4), there is a positive integer $k(1)$ such that ord $\left(p, \mathscr{W}_{1, k(1)}\right)$ is finite. Let $\left\{W_{1}, \cdots, W_{t_{1}}\right\}$ be all elements of $\mathscr{W}_{1, k(1)}$ which contain $p$ and intersect $M$. Such elements of $\mathscr{W}_{1, k(1)}$ exist since $p \in V_{1, k(1)}$. By (1), for $r=1, \cdots, t_{1}$, there is some $x_{r}$ in $M$ such that $W_{r} \subseteq H\left(1, x_{r}\right)$. Let $T_{1}=\left\{x_{1}, \cdots, x_{t_{1}}\right\}$ and let $j(1)=1$.

Now let $j(2)=j(1)+k(1)+1$, and by (4) pick a positive integer $k(2)$ such that ord $\left(p, \mathscr{W}_{j(2), k(2)}\right)$ is finite. Let $\left\{W_{1}^{\prime}, \cdots, W_{t_{2}}^{\prime}\right\}$ be all elements of $\mathscr{W}_{j(2), k(2)}$ which contain $p$ and intersect $M$. Such sets exist since $p \in V_{j(2), k(2)}$. By (1), for $r=1, \cdots, t_{2}$, there is some $x_{r}^{\prime}$ in $M$ such that $W_{r}^{\prime} \leqq H\left(j(2), x_{r}^{\prime}\right)$. By (3), for $r=1, \cdots, t_{2}, \overline{H\left(j(2), x_{r}^{\prime}\right)}$ is contained in some element of $\mathscr{W}_{1, k(1)}$. It easily follows that $\overline{H\left(j(2), x_{r}^{\prime}\right)} \leqq H\left(1, x_{s}\right)$ for some $x_{s}$ in $T_{1}$. Let $T_{2}=\left\{x_{r}^{\prime}: 1 \leqq r \leqq t_{2}\right\}$.

Continuing this process, we obtain a strictly increasing sequence of positive integers $1=j(1)<j(2)<\cdots<j(n)<\cdots$, and for each $n$ a nonempty finite subset $T_{n}$ of $M$, such that the following hold for all $n$ :

(5) if $x \in T_{n}$, then $p \in H(j(n), x)$;

(6) if $x \in T_{n+1}$, then $\overline{H(j(n+1), x)} \subseteq H(j(n), y)$ for some $y$ in $T_{n}$.

For $n=1,2, \cdots$ let $\mathscr{H}_{n}=\left\{H(j(n), x): x\right.$ in $\left.T_{n}\right\}$. Then by Lemma 3.1, there is a sequence $\left\langle x_{n}\right\rangle$ in $M$ such that $p \in H\left(j(n), x_{n}\right)$ and $\overline{H\left(j(n+1), x_{n+1}\right)} \subseteq H\left(j(n), x_{n}\right)$ for all $n$. Now by $(2), H\left(j(n), x_{n}\right) \subseteq$ $l\left(j(n), x_{n}\right) \subseteq l\left(n, x_{n}\right)$, so $p \in l\left(n, x_{n}\right), n=1,2, \cdots$. Since $l$ is a $\beta$-function, $\left\langle x_{n}\right\rangle$ has a cluster point, say $q$. Clearly $q \in M$, and it is easy to check that $q \in H\left(j(n), x_{n}\right), n=1,2, \cdots$. (Recall that $x_{n} \in H\left(j(n), x_{n}\right.$ ) by (2), and that $\overline{H\left(j(n+1), x_{n+1}\right)} \subseteq H\left(j(n), x_{n}\right)$.) Now $p \notin M$, so $p \neq q$. Hence there is some $n_{0}$ such that $\operatorname{st}\left(q, \mathscr{G}_{n_{0}}\right) \neq \phi$ and $p \notin \operatorname{st}\left(q, \mathscr{G}_{n_{0}}\right)$. Choose $n \geqq n_{0}$ such that $x_{n} \in \operatorname{st}\left(q, \mathscr{G}_{n_{0}}\right)$. Since $x_{n} \in \mathscr{G}_{n_{0}}^{*}$, there is some 
$i_{0} \leqq n_{0}$ such that $n_{0}=m\left(i_{0}, x_{n}\right)$. Note that $G\left(i_{0}, x_{n}\right) \in \mathscr{G}_{m\left(i_{0}, x_{n}\right)}=\mathscr{G}_{n_{0}}$ and that $i_{0} \leqq n$. Now $\{p, q\} \subseteq H\left(j(n), x_{n}\right) \subseteq \bigcap_{i=1}^{j(n)} G\left(i, x_{n}\right)$, so both $p$ and $q$ belong to $G\left(i_{0}, x_{n}\right)$. Thus $p \in \operatorname{st}\left(q, \mathscr{G}_{n_{0}}\right)$, a contradiction. Hence $p \in M$, and so the proof that $M$ is a $G_{\delta}$ is complete.

CoRollaRY 3.3. Every regular $\theta$-refinable $\Sigma^{\sharp}$-space with a quasi$G_{\delta}$-diagonal is a $\sigma$-space.

Proof. Let $X$ be a regular $\theta$-refinable $\Sigma^{\sharp}$-space with a quasi- $G_{j^{-}}$ diagonal. Now every $\Sigma^{\sharp}$-space is a $\beta$-space (see [34]), and so $X$ is semi-stratifiable by Theorem 3.2. Since every regular semi-stratifiable $\Sigma^{\sharp}$-space is a $\sigma$-space (see [57]), the proof is complete.

Corollary 3.4. (Bennett and Berney [11].) Every regular $\theta$ refinable $\beta$-space with a quasi-development is developable.

Proof. By Theorem 3.2, such a space is semi-stratifiable. Since every semi-stratifiable space with a quasi-development is developable (see [10]), the proof is complete.

CoRollaRY 3.5. Every regular $\theta$-refinable ws-space with a quasi$G_{\tilde{o}}$-diagonal is developable.

Proof. By Theorem 3.2, such a space is semi-stratifiable. Since every regular semi-stratifiable $w \Delta$-space is developable (see [22]), the proof is complete.

CoRollary 3.6. Every paracompact ws-space with a quasi-G $G_{\dot{o}^{-}}$ diagonal is metrizable.

Proof. By Corollary 3.5, such a space is developable. Since every paracompact developable space is metrizable [12], the proof is complete.

COROLLARY 3.7. Every paracompact ws-space with a $\theta$-separating cover is metrizable.

Proof. Let $X$ be a paracompact $w \Delta$-space, let $\mathscr{G}_{1}, \mathscr{G}_{2}, \cdots$ be a $\theta$-separating cover of $X$. (Recall that each $\mathscr{G}_{n}$ is an open collection and that given any two distinct points $p$ and $q$ in $X$, there is some $n$ such that ord $\left(p, \mathscr{G}_{n}\right)$ is finite and there is some $G$ in $\mathscr{G}_{n}$ such that $p \in G, q \notin G$. See [31].) For each pair of positive integers $n$ and $k$ let $\mathscr{H}_{n k}=\left\{H: H \neq \phi, H=\bigcap_{i=1}^{k} G_{i}, G_{1}, \cdots, G_{k}\right.$ distinct elements of $\left.\mathscr{G}_{n}\right\}$. Then $\left\{\mathscr{C}_{n k}: n, k\right.$ in $\left.N\right\}$ is a quasi-G $G_{\delta}$-diagonal for $X$, so by Corollary $3.6 X$ is metrizable. 
CoRollaRY 3.8. (Bennett and Berney [11].) Every paracompact $\beta$-space with a quasi-development is metrizable.

4. Hereditarily CCC spaces. In [3] Arhangel'skii proved that every regular space which is hereditarily a Lindelöf $p$-space has a countable base. In [33] the author obtained an analogue of Arhangel'skil's theorem by showing that every regular space which is hereditarily a CCC strong $\Sigma$-space has a countable net. These two results suggest numerous questions concerning the metrizability of hereditarily CCC spaces. The following problem seems particularily interesting.

Problem 4.1. Does every regular space which is hereditarily a CCC $w \Delta$-space have a countable base?

In this section we show that the answer to 4.1 is "yes" under the additional assumption that the space has a point-countable separating open cover. We begin with a lemma due to Arhangel'skiil [3]. Since it is not clear in Arhangel'skil's paper that this lemma is independent of the continuum hypothesis, we sketch a proof here. The main idea of the proof, of course, is Arhangel'skily's.

Lemma 4.2. (Arhangel'skil) Let $X$ be a regular CCC space which is hereditarily a q-space. Then $X$ is first countable.

Proof. Let $p$ be a point of $X$, and let us show that $p$ has a countable fundamental system of neighborhoods. Let $\mathscr{Y}$ be a maximal mutually disjoint collection of nonempty open sets in $X$ such that $p \notin \bar{V}$ for all $V$ in $\mathscr{V}$. Since $X$ satisfies the CCC, $\mathscr{V}$ is countable, say $\mathscr{V}=\left\{V_{n}: n=1,2, \cdots\right\}$. Let $Z=\{p\} \cup\left(\bigcup_{n=1}^{\infty} V_{n}\right)$. By the maximality of $\mathscr{Y}, Z$ is a dense subset of $X$. Now $Z$ is a regular $q$-space, and $p$ is a $G_{\delta}$ in $Z\left(\{p\}=\bigcap_{n=1}^{\infty}\left(Z-\bar{V}_{n}\right)\right)$, so by a result due to Lutzer [40] $p$ has a countable fundamental system of neighborhoods in $Z$. Since $Z$ is dense in $X$, it follows (see p. 10 of [39]) that $p$ has a countable fundamental system of neighborhoods in $X$.

Theorem 4.3. A regular space has a countable base if and only if it has a point-countable separating open cover and is hereditarily a CCC ws-space.

Proof. Let $X$ be a regular space. If $X$ has a countable base, the result is clear. Assume, then, that $X$ has a point-countable separating open cover $\mathscr{S}$ and is hereditarily a CCC $w \Delta$-space. First we show that $X$ is separable. (The technique used here is due to Ponomarev [53].) Suppose $X$ is not separable. Then there is a subset 
$Y=\left\{x_{\sigma}: 0 \leqq \sigma<\omega_{1}\right\}$ of $X$ such that $x_{\sigma} \notin\left\{x_{\tau}: \tau<\sigma\right\}^{-}$for all $\sigma<\omega_{1}$. Now by Lemma 4.2, $X$ is first countable, and so for each $\sigma<\omega_{1}$ there is a countable fundamental system of open neighborhoods $\{V(\sigma, n): n=$ $1,2, \cdots\}$ of $x_{\sigma}$ such that for all $n, V(\sigma, n) \cap\left\{x_{\varepsilon}: \tau<\sigma\right\}=\phi$. Let $\mathscr{V}=$ $\left\{V(\sigma, n) \cap Y: 0 \leqq \sigma<\omega_{1}, n=1,2, \cdots\right\}$, and note that $\mathscr{V}$ is a pointcountable base for $Y$. Since $Y$ is $\aleph_{1}$-compact, it follows from a result of Aquaro [2] that $Y$ is Lindelöf. (Also, see Lemma 3.6 of [33].) Thus $Y$ is a Lindelöf $w \Delta$-space with a point-countable base and hence has a countable base [53]. This contradicts the fact that $Y$ is not separable.

Since $X$ is separable, the point-countable separating open cover $\mathscr{S}$ is actually countable. Now every regular $w \Delta$-space with a $\sigma$-point finite separating open cover is developable [31]. Thus $X$ is a developable $\aleph_{1}$-compact space and hence has a countable base [38].

REMARK 4.4. The above argument can be modified to prove the following result. Every regular space which is hereditarily a quasicomplete CCC space is hereditarily separable.

REMARK 4.5. Gittings [25] has recently proved that every regular quasi-complete space with a $G_{\delta}$-diagonal is a $p$-space. His argument also shows that such a space is hereditarily a $p$-space. By Remark 4.4, then, we obtain this result. Every regular quasi-complete space with a $G_{-}$-diagonal which is hereditarily a CCC space is hereditarily separable. It should be noted that Burke [17] has constructed a regular $p$-space with a $G_{j}$-diagonal (hence a hereditarily $p$-space) which is not developable.

Problem 4.6. Does every regular $w \Delta$-space with a $G_{\delta}$-diagonal which hereditarily satisfies the CCC have a countable base?

5. Baire spaces satisfying the CCC. In [55] Šapirovskil showed that every $\widehat{C}$ ech complete space has a dense subspace which is a para-

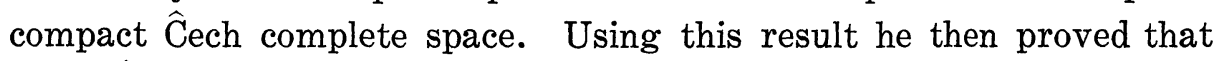
every $\widehat{C}$ ech complete space with a point-countable base which satisfies the CCC has a countable base. In this section we obtain similar results.

Lemma 5.1. Let $X$ be a regular space, let $\mathscr{G}_{1}, \mathscr{G}_{2}, \cdots$ be a pluming for $X$ such that each $\mathscr{G}_{n}$ is a locally finite collection. Then $X$ is paracompact.

Proof. Let $\mathscr{R}$ be an open cover of $X$, let $\mathscr{R}^{\prime}$ be all finite unions 
of elements of $\mathscr{R}$. It suffices to show that $\mathscr{R}^{\prime}$ has a $\sigma$-locally finite open refinement [42]. For $n=1,2, \cdots$ let $\mathscr{C}_{n}=\Lambda_{i=1}^{n} \mathscr{G}_{i}$, and note that $\mathscr{H}_{n}$ is a locally finite open cover of $X$. For $n=1,2, \cdots$ let

$\mathscr{L}_{n}=\left\{H\right.$ in $\mathscr{K}_{n}: H$ is contained in some element of $\left.\mathscr{R}^{\prime}\right\}$.

To complete the proof it suffices to show that $\bigcup_{n=1}^{\infty} \mathscr{L}_{n}$ covers $X$. Let $p \in X$, and for $n=1,2, \cdots$ let $p \in G_{n} \in \mathscr{G}_{n}$. Now $C(p)=\bigcap_{n=1}^{\infty} \bar{G}_{n}$ is compact, so there is some $R$ in $\mathscr{R}^{\prime}$ such that $C(p) \subseteq R$. Choose $n$ in $N$ such that $\bigcap_{i=1}^{n} \bar{G}_{\imath} \subseteq R$. Then $H=\bigcap_{i=1}^{n} G_{i}$ is an element of $\mathscr{L}_{n}$ such that $p \in H$.

Lemma 5.2. Every regular p-space which is a Baire space has a dense subspace which is a paracompact p-space.

Proof. Let $X$ be a regular Baire space, and let $\mathscr{G}_{1}, \mathscr{G}_{2}, \cdots$ be a pluming for $X$. We may assume that each $\mathscr{G}_{n}$ is a base for $X$. (See 4.2 in [36].) With the aid of the axiom of choice, construct a sequence $\mathscr{L}_{1}, \mathscr{C}_{2}, \cdots$ of open collections satisfying these conditions.

(1) $\mathscr{H}_{n}$ is a mutually disjoint subcollection of $\mathscr{G}_{n}$;

(2) $V_{n}=\cup\left\{H: H\right.$ in $\left.\mathscr{C}_{n}\right\}$ is a dense subset of $X$;

(3) for all $n$, if $H \in \mathscr{H}_{n+1}$, then $\bar{H} \subseteq H^{\prime}$ for some $H^{\prime}$ in $\mathscr{H}_{n}$.

The technique for constructing such a sequence is well known; see, e.g., [8], [44], or [54]. Let $D=\bigcap_{n=1}^{\infty} V_{n}$, and note that $D$ is a dense subset of $X$ since $X$ is a Baire space. For $n=1,2, \cdots$ let $\mathscr{L}_{n}=$ $\left\{H \cap D: H\right.$ in $\left.\mathscr{H}_{n}\right\}$. It is clear that $\mathscr{L}_{1}, \mathscr{L}_{2}, \ldots$ is a sequence of locally finite (in fact discrete) open covers of $D$, and so to complete the proof it suffices to show that $\mathscr{L}_{1}, \mathscr{L}_{2}, \cdots$ is a pluming for $D$. (Recall Lemma 5.1.)

Let $p \in D$, for $n=1,2, \cdots$ let $p \in L_{n}=H_{n} \cap D$, where $H_{n} \in \mathscr{H}_{n}$, and let $C(p)=\bigcap_{n=1}^{\infty} c l_{D}\left(L_{n}\right)$. We must show that (a) $C(p)$ is compact; (b) $\left\{\bigcap_{i=1}^{n} c l_{D}\left(L_{\imath}\right): n=1,2, \cdots\right\}$ is a "base" for $C(p)$. Observe that $p \in H_{n} \in \mathscr{G}_{n}, \quad n=1,2, \cdots$ and so $C^{*}(p)=\bigcap_{n=1}^{\infty} \bar{H}_{n}$ is compact and $\left\{\bigcap_{i=1}^{n} \bar{H}_{2}: n=1,2, \cdots\right\}$ is a "base" for $C^{*}(p)$. We are going to show that $C(p)=C^{*}(p)$, from which both (a) and (b) easily follow.

Clearly $C(p) \subseteq C^{*}(p)$; so let $q \in C^{*}(p)$, and let us show that $q \in C(p)$. First, $q \in D$, since for all $n, q \in \bar{H}_{n+1} \subseteq H_{n} \subseteq V_{n}$. (That $\bar{H}_{n+1} \subseteq H_{n}$ follows from (1), (3), and the fact that $p \in\left(H_{n} \cap H_{n+1}\right)$.) To show that $q \in$ $c l_{D}\left(L_{n}\right)$, consider an arbitrary open set $R$ in $X$ containing $q$. Now $q \in \bar{H}_{n}$, so $R \cap H_{n} \neq \dot{\phi}$. Since $D$ is dense, $\left(R \cap H_{n}\right) \cap D \neq \phi$. But $L_{n}=$ $H_{n} \cap D$, so $R \cap L_{n} \neq \phi$.

LEMMA 5.3. Every regular quasi-complete space with a pointcountable separating open cover is a p-space. 
Proof. Let $X$ be a regular space with a point-countable separating open cover, let $\mathscr{G}_{1}, \mathscr{G}_{2}, \cdots$ be a quasi-complete sequence for $X$. For $n=1,2, \cdots$ let $\mathscr{H}_{n}$ be an open cover of $X$ such that $\left\{\bar{H}: H\right.$ in $\left.\mathscr{H}_{n}\right\}$ refines $\mathscr{G}_{n}$. Let $p \in H_{n} \in \mathscr{H}_{n}, n=1,2, \cdots$. Using the fact that $\mathscr{G}_{1}, \mathscr{G}_{2}, \ldots$ is a quasi-complete sequence, one can easily check that (a) $C(p)=\bigcap_{n=1}^{\infty} \bar{H}_{n}$ is countably compact and (b) $\left\{\bigcap_{i=1}^{n} \bar{H}_{i}: n=1,2, \cdots\right\}$ is a "base" for $C(p)$. Since $C(p)$ is a regular countably compact space with a point-countable separating open cover, it is compact [7]. Hence $\mathscr{H}_{1}, \mathscr{H}_{2}, \cdots$ is a pluming for $X$.

THEOREM 5.4. Every regular wL-space with a point-countable separating open cover which is a Baire space and hereditarily satisfies the CCC has a countable base.

Proof. Let $X$ be a space satisfying the hypotheses of the theorem. By Lemma 5.3, $X$ is a $p$-space. Hence, by Lemma 5.2, $X$ has a dense subspace $D$ which is a paracompact $p$-space. Now $D$ also has a pointcountable separating open cover, and so $D$ is metrizable [47]. Using the denseness and the metrizability of $D$, together with the fact that $X$ satisfies the CCC, one can show that $X$ is separable. From this it follows that $X$ has a countable separating open cover. Now every regular $w \Delta$-space with a $\sigma$-point finite separating open cover is developable [31]. Thus $X$ is a developable $\boldsymbol{\aleph}_{1}$-compact space and hence has a countable base [38].

REMARK 5.5. Comparing Theorems 4.3 and 5.4, we see that "hereditarily $w \Delta$-space" in 4.3 can be weakened to " $w \Delta$-space", provided the assumption "Baire space" is added. This suggests the following problem.

Problem 5.6. Does every regular $w \Delta$-space with a point-countable separating open cover which hereditarily satisfies the CCC have a countable base?

TheOREm 5.7. Let $X$ be a regular quasi-complete Baire space which satisfies the CCC. If $X$ has a $G_{\dot{o}}$-diagonal, or a point-countable separating open cover, then $X$ is separable.

Proof. If $X$ has a $G_{i}$-diagonal, then by a result of Gittings [25], $X$ is a $p$-space. And, if $X$ has a point-countable separating open cover, then $X$ is a $p$-space by Lemma 5.3. In either case, then, by Lemma 5.2 there is a dense subspace $D$ of $X$ which is a paracompact $p$-space. Since $D$ has a $G_{j}$-diagonal or a point-countable separating open cover, $D$ is metrizable (see [13], [51], [47]). Now $D$ is dense in $X$ and $X$ 
satisfies the CCC, so $D$ also satisfies the CCC. Thus $D$ is separable, from which it follows that $X$ is separable.

REMARK 5.8. From Theorem 5.7 we obtain the following slight generalization of the result of Sapirovskir mentioned at the beginning of this section. Every regular quasi-complete Baire space with a point-countable base which satisfies the CCC has a countable base. It should be noted that the "Baire space" hypothesis in this result and Theorem 5.7 is not superfluous. Indeed, Pixley and Roy [52] have constructed a regular developable space with a $\sigma$-point finite base which satisfies the CCC but is not separable.

6. A metrization theorem for Moore spaces. In this section we extend two theorems of Traylor on the metrizability of Moore spaces. (See Theorems 4 and 5 of [63].) This new result should be compared with a theorem of Borges [14], which states that every locally connected, locally peripherally compact paracompact space with a $G_{\hat{o}^{-}}$ diagonal is metrizable.

THEOREM 6.1. Let $X$ be a locally connected, locally peripherally separable meta-Lindelöf Moore space. Then $X$ is metrizable.

Proof. It suffices to show that $X$ is paracompact [12]. Let $\mathscr{C}=$ $\left\{C_{\alpha}: \alpha\right.$ in $\left.A\right\}$ be the collection of all distinct components of $X$. Thus, each $C_{\alpha}$ is a closed, connected subset of $X$, and $\alpha \neq \beta$ implies $C_{\alpha} \cap$ $C_{\beta}=\phi$. Since $X$ is locally connected, each $C_{\alpha}$ is also an open subset of $X$. It is easy to check that each $C_{\alpha}$ is a connected, locally connected, locally peripherally separable meta-Lindelöf space, and so by a result of Grace and Heath [26], each $C_{\alpha}$ is Lindelöf. Now any regular space which is the union of a disjoint collection of open sets, each of which is Lindelöf, is paracompact. This completes the proof.

7. Metrizability of countable unions. Let $X$ be a regular space, and suppose that $X=\bigcup_{n=1}^{\infty} M_{n}$, where each $M_{n}$ is a metrizable subset of $X$. The following are known conditions under which $X$ itself is metrizable:

(1) $X$ is locally countably compact and each $M_{n}$ is separable (Smirnov [60]);

(2) $X$ is collectionwise normal and locally countably compact, and each $M_{n}$ is closed (Stone [62]);

(3) $X$ is a collectionwise normal $p$-space, $M_{1}$ is separable, and $M_{n}$ is closed for $n \geqq 2$ (Čoban [20]);

(4) each $M_{n}$ is locally dense, and $X$ is one of the following: (i) separable; (ii) locally compact; (iii) $\sigma$-compact (Corson-Michael [21]). 
\{Recall that a subset of $X$ is locally dense if it is a dense subset of an open subset of $X$. This definition, due to Corson and Michael, is a clever device for considering subsets which are either open or dense.\}

In this section we obtain generalizations of these results. In particular, Theorem 7.1 unifies and generalizes (1), (2), and (3), while Theorem 7.4 extends (i) and (iii) of (4).

TheOREM 7.1. Let $X$ be a collectionwise normal space which is locally a quasi-complete space, and suppose that $X=\bigcup_{n=1}^{\infty} M_{n}$, where each $M_{n}$ is metrizable and either separable or closed. Then $X$ is metrizable.

Proof. If $M_{n}$ is separable, then it has a countable base and hence a countable net. On the other hand, if $M_{n}$ is closed, then there is a $\sigma$-locally finite collection in $X$ which is a net for $M_{n}$. It easily follows that $X$ has a $\sigma$-locally finite net, and hence is a $\sigma$-space. In particular, then, $X$ is semi-stratifiable and subparacompact [22]. Now every regular semi-stratifiable quasi-complete space is developable [22], and so $X$ is locally a developable space. Since every subparacompact locally developable space is developable [15], we see that $X$ is a collectionwise normal developable space and thus metrizable [12].

REMARK 7.2. It is clear that (2) and (3) follow from Theorem 7.1. To see that (1) also follows from 7.1, note that a countable union of separable metrizable spaces is Lindelof and that every regular Lindelöf space is collectionwise normal. As the following example shows, the hypothesis that $X$ is locally a quasi-complete space is not superfluous.

EXAMPLE 7.3. A nonmetrizable, paracompact semi-metric space which is a countable union of closed separable metrizable subsets. The space $S$ described by Heath in [27] has the required properties.

THeOREM 7.4. Let $X$ be a regular space such that $X=\bigcup_{n=1}^{\infty} M_{n}$, where each $M_{n}$ is a locally dense, metrizable subset of $X$. Then $X$ is metrizable if it satisfies any of the following:

(a) $X$ is locally a CCC space;

(b) $X$ is a $w N$-space;

(c) $X$ is a normal $\beta$-space;

(d) $X$ is a normal countably metacompact p-space.

Proof. First we show that $X$ has a $\sigma$-disjoint base. For $n=$ $1,2, \cdots$ let $R_{n}$ be an open subset of $X$ such that $M_{n}$ is a dense subset of $R_{n}$. Since $M_{n}$ is metrizable, it has a $\sigma$-disjoint base $\mathscr{B}_{n}=$ 
$\bigcup_{k=1}^{\infty} \mathscr{B}_{n, k}$, where $\mathscr{B}_{n, k}=\left\{B_{\alpha}: \alpha\right.$ in $\left.A_{n, k}\right\}$ is a disjoint open collection in $M_{n}$. For each $\alpha$ in $A_{n, k}$ let $G_{\alpha}$ be an open subset of $R_{n}$ such that $G_{\alpha} \cap M_{n}=B_{\alpha}$, and let $\mathscr{G}_{n, k}=\left\{G_{\alpha}: \alpha\right.$ in $\left.A_{n, k}\right\}$. It is easy to check that $\mathscr{G}_{n, k}$ is a disjoint open collection in $X$. To see that $\left\{\mathscr{G}_{n, k}: n, k\right.$ in $\left.N\right\}$ is a base for $X$, let $p \in X$ and let $U$ be an open neighborhood of $p$. Let $V$ be an open neighborhood of $p$ such that $\bar{V} \cong U$, and choose $n$ in $N$ such that $p \in M_{n}$. For some $\alpha$ in $A_{n, k}, p \in B_{\alpha} \subseteq V \cap M_{n}$. Now $G_{\alpha} \subseteq \bar{B}_{\alpha}$ since $M_{n}$ is dense in $R_{n}$, so $p \in G_{\alpha} \subseteq U$.

As consequences of the fact that $X$ has a $\sigma$-disjoint base, we note that $X$ is screenable and is a $\gamma$-space.

To prove (a), assume that $X$ is locally a CCC space. Since $X$ has a $\sigma$-disjoint base, it easily follows that $X$ is locally metrizable and locally separable. Now it is easy to check that every open cover of a locally separable screenable (in fact meta-Lindelöf) space has a starcountable open refinement. It follows that $X$ is paracompact. (See [58] or p. 229 of [23].) Since every paracompact, locally metrizable space is metrizable [59], the proof of (a) is complete.

To prove (b), assume that $X$ is a $w N$-space. Since $X$ has been observed to be a $\gamma$-space, it follows that $X$ is metrizable [32].

To prove (c), assume that $X$ is a normal, $\beta$-space. The metrizability of $X$ follows from the fact that every $T_{1}, \beta, \gamma$-space is developable [32] and that every normal screenable developable space is metrizable [12].

To prove (d), assume that $X$ is a normal, countably metacompact $p$-space. By (c), it suffices to show that $X$ is a $\beta$-space. This follows from the fact that every countably metacompact screenable space is metacompact and that every metacompact $p$-space is a $\beta$-space [16].

REMARK 7.5. Note that (i) of (4) follows from (a) of Theorem 7.4, while (iii) of (4) follows from (c) of 7.4. In addition, (b) of 7.4 generalizes Theorem 5.1 in [37]. As the following example shows, the assumptions in (a), (b), (c), and (d) are not superfluous.

EXAMPLE 7.6. A nonmetrizable completely regular developable space which is the union of two open metrizable subsets. Consider the space $S$ described by Heath in [28, Example 2]. The points of $S$ are all points of the plane on or above the $x$-axis, and $S$ (equipped with the topology described in [28]) is a completely regular nonnormal screenable developable space. Let $U$ be all rational points on the $x$ axis together with all points of the plane above the $x$-axis, and let $V$ be all irrational points on the $x$-axis together with all points of the plane above the $x$-axis. Then $S=U \cup V$, and $U$ and $V$ are open metrizable subsets of $S$. 
In [21] Corson and Michael prove that every normal Ĉech complete space which is the union of two locally dense metrizable subsets is metrizable. They also ask if the restriction to just two sets is really necessary. We now show that their result holds for any finite union, and that "Ĉech complete" can be weakened to " $p$-space".

LEMma 7.7. Let $M$ be a locally dense, countably paracompact subset of a topological space $X$, let $\left\{U_{n}: n=1,2, \cdots\right\}$ be a countable open collection in $X$ which covers $M$. Then there is a point-finite open collection $\left\{W_{n}: n=1,2, \cdots\right\}$ in $X$ which covers $M$ such that $W_{n} \subseteq U_{n}, n=1,2, \cdots$.

Proof. Let $R$ be an open set in $X$ such that $M$ is a dense subset of $R$. Since $M$ is countably paracompact, there is a cover $\left\{V_{n}: n=\right.$ $1,2, \cdots\}$ of $M$, locally finite and open in $M$, such that $V_{n} \subseteq U_{n} \cap M$, $n=1,2, \cdots$. For each $n$ let $G_{n}$ be an open subset of $R$ such that $G_{n} \cap M=V_{n}$, and let

$$
L=\left\{p \in R:\left\{G_{n}: n=1,2, \cdots\right\} \text { is locally finite at } p\right\} \text {. }
$$

Clearly $L$ is open, and $M \subseteq L$ since $M$ is dense in $R$. For $n=1,2, \cdots$ let $W_{n}=L \cap G_{n} \cap U_{n}$. Then $\left\{W_{n}: n=1,2, \cdots\right\}$ is the desired pointfinite open collection.

THEOREM 7.8. Let $X$ be a normal p-space which is covered by a finite number of locally dense, metrizable subsets. Then $X$ is metrizable.

Proof. By Theorem 7.4, part (d), it suffices to show that $X$ is countably metacompact. But this follows immediately from Lemma 7.7 .

Added in proof. The notion of a quasi- $G_{\delta}$-diagonal has been studied by other topologists. In particular, Lutzer [Dissertationes Math. 89 (1971)] has proved that a LOTS is quasi-developable if and only if it has a quasi- $G_{\dot{\delta}}$-diagonal, and Shiraki [Proc. Japan Acad. 47 (1971), suppl. II, 1036-1041] has proved that every regular, metacompact, $w \Delta$-space with a quasi- $G_{\dot{0}}$-diagonal has a uniform base. A. Okuyama, in a private communication, has answered Problem 5.6 affirmatively under the assumption of the continuum hypothesis.

\section{REFERENCES}

1. P. S. Alexandroff and P. Urysohn, Une condition nessaire et suffisante pour qu'une class (L) sait une class (D), C. R. Acad. Sci. Paris, 177 (1923), 1274-1277. 
2. G. Aquaro, Point-countable open coverings in countably compact spaces, General Topology and Its Relations to Modern Analysis and Algebra II, Academia, Prague (1966), 39-41.

3. A. V. Arhangel'skiř, On hereditary properties, General Topology and Its Applications, 3 (1973), 39-46.

4. - On a class of spaces containing all metric and all locally bicompact spaces, Amer. Math. Soc. Transl., 2 (1970), 1-39.

5. — Mappings and spaces, Russian Math. Surveys, 21 (1966), 115-162.

6. —, An addition theorem for the weight of spaces lying in bicompacta, Dokl. Akad. Nauk SSSR, 126 (1959), 239-241.

7. A. V. Arhangel'skiY and V. Proizvolov, On the connection between the point-cardinality of systems of subsets of a bicompact set and its weight, Vestnik Moskov. Univ. Ser. I Mat. Meh., 21 (1966), 75-77.

8. S. Armentrout, Completing Moore spaces, Proceedings of the Arizona State U. Topology Conference, (1967), 22-35.

9. H. R. Bennett, $A$ note on the metrizability of $M$-spaces, Proc. Japan Acad., 45 (1969), 6-9.

10. - On quasi-developable spaces, General Topology and Its Applications, 1 (1971), 253-262.

11. H. R. Bennett and E. S. Berney, On certain generalizations of developable spaces, General Topology and its Applications, 4 (1974), 43-50.

12. R. H. Bing, Metrization of topological spaces, Canad. J. Math., 3 (1951), 175-186.

13. C. J. R. Borges, On stratifiable spaces, Pacific J. Math., 17 (1966), 1-16.

14. - On metrizability of topological spaces, Canad. J. Math., 20 (1968), 795804.

15. D. K. Burke, On subparacompact spaces, Proc. Amer. Math. Soc., 23 (1969), 655-663.

16. —, On p-spaces and w4-spaces, Pacific J. Math., 35 (1970), 285-296.

17. — A nondevelopable locally compact Hausdorff space with a Go-diagonal, General Topology and Its Applications, 2 (1972), 287-291.

18. J. G. Ceder, Some generalizations of metric spaces, Pacific J. Math., 11 (1961), $105-125$.

19. M. M. Čoban, Mappings of metric spaces, Soviet Math. Dokl., 10 (1969), 258-260. 20. - Some metrization theorems for p-spaces, Soviet Math. Dokl., 8 (1967), 561-563.

21. H. H. Corson and E. Michael, Metrizability of certain countable unions, Illinois J. Math., 8 (1964), 351-360.

22. G. Creede, Concerning semi-stratifiable spaces, Pacific J. Math., 32 (1970), 47-54.

23. R. Engelking, Outline of General Topology, Interscience Publishers, 1968.

24. V. V. Filippov, On feathered paracompacta, Soviet Math. Dokl., 9 (1968), 161-164.

25. R. F. Gittings, Concerning quasi-complete spaces, Notices Amer. Math. Soc., 20 (1973), A-511.

26. E. E. Grace and R. W. Heath, Separability and metrizability in pointwise paracompact Moore spaces, Duke Math. J., 31 (1964), 603-610.

27. R. W. Heath, A paracompact semi-metric space which is not an $M_{3}$-space, Proc. Amer. Math. Soc., 17 (1966), 868-870.

28. - Screenability, pointwise paracompactness, and metrization of Moore spaces, Canad. J. Math., 16 (1964), 763-770.

29. - On open mappings and certain spaces satisfying the first countability axiom, Fund. Math., 57 (1965), 91-96.

30. - Arc-wise connectedness in semi-metric spaces, Pacific J. Math., 12 (1962), 1301-1319.

31. R. E. Hodel, Moore spaces and wd-spaces, Pacific J. Math., 38 (1971), 641-652.

32. - Spaces defined by sequences of open covers which guarantee that certain 
sequences have cluster points, Duke Math. J., 39 (1972), 253-263.

33. R. E. Hodel, On a theorem of Arhangel'ski乞 concerning Lindelöf p-spaces, Topology Conference, Virginia Polytechnic Institute and State University, Lecture Notes in Mathematics, 375 (1974), 120-136.

34. - - Spaces characterized by sequences of covers which guarantee that certain sequences have cluster points, Proceedings of the University of Houston Point Set Topology Conference, (1971), 105-114.

35. - Some results in metrization theory, 1950-72, Topology Conference, Virginia Polytechnic Institute and State University, Lecture Notes in Mathematics, 375 (1974), 120-136.

36. - Extensions of metrization theorems to higher cardinality, to appear in Fund. Math.

37. T. Ishii and T. Shiraki, Some properties of $w M$-spaces, Proc. Japan Acad., 47 (1971), 167-172.

38. F. B. Jones, Concerning normal and completely normal spaces, Bull. Amer. Math. Soc., 43 (1937), 671-677.

39. I. Juhász, Cardinal Functions in Topology, Mathematical Centre, Amsterdam, 1971.

40. D. J. Lutzer, Semimetrizable and stratifiable spaces, General Topology and Its Applications, 1 (1971), 43-48.

41. E. Michael, $A$ note on closed maps and compact sets, Israel J. Math., 2 (1964), $173-176$.

42. - - A note on paracompact spaces, Proc. Amer. Math. Soc., 4 (1953), 831-838.

43. - On Nagami's $\Sigma$-spaces and some related matters, Proceedings of the

Washington State University Conference on General Topology, (1970), 13-19.

44. R. L. Moore, Concerning separability, Proc. Nat. Acad. Sci. U.S.A., 28 (1942), $56-58$.

45. — Foundations of Point Set Theory, Amer. Math. Soc. Colloquium Publ. 13, rev. ed. 1962.

46. K. Nagami, $\Sigma$-spaces, Fund. Math., 65 (1969), 169-192.

47. J. Nagata, A note on Filippov's theorem, Proc. Japan Acad., 45 (1969), 30-33.

48. - A contribution to the theory of metrization, J. Inst. Polytech. Osaka City Univ. Ser. A, Math., 8 (1957), 185-192.

49. On a necessary and sufficient condition of metrizability, J. Inst. Polytech. Osaka City Univ. Ser. A, Math., 1 (1950), 93-100.

50. A. Okuyama, Some generalizations of metric spaces, their metrization theorems and product theorems, Sci. Rep. Tokyo Kyoiku Daigaku, Ser. A, 9 (1967), 236-254.

51. - On metrizability of M-spaces, Proc. Japan Acad., 40 (1964), 176-179.

52. C. Pixley and P. Roy, Uncompletable Moore spaces, Proceedings of the Auburn Topology Conference, (1969), 75-85.

53. V. I. Ponomarev, Metrizability of a finally compact p-space with a point-countable base, Soviet Math. Dokl., 8 (1967), 765-768.

54. G. M. Reed, Concerning first countable spaces, II, Duke Math. J., 40 (1973), 677-682.

55. B. Šapirovskiř, On separability and metrizability of spaces with Souslin's condition, Soviet Math. Dokl., 13 (1972), 1633-1638.

56. F. Siwiec, On defining spaces by a weak base, Pacific J. Math., 52 (1974), 233-245.

57. F. Siwiec and J. Nagata, $A$ note on nets and metrization, Proc. Japan Acad., 44 (1968), 623-627.

58. Yu. M. Smirnov, On strongly paracompact spaces, Izv. Akad. Nauk SSSR Ser. Mat., 20 (1956), 253-274.

59. - A necessary and sufficient condition for metrizability of a topological space, Doklady Akad. Nauk SSSR (N.S.), 77 (1951), 197-200.

60. - On the metrizability of bicompacts decomposable into a sum of sets with countable basis, Fund. Math., 43 (1956), 387-393. 
61. Mun-Gu Sohn and Bong Dae Choi, On quasi-complete spaces, Kyungpook Math. J., 12 (1972), 225-228.

62. A. H. Stone, Metrizability of unions of spaces, Proc. Amer. Math. Soc., 10 (1959), 361-366.

63. D. R. Traylor, Concerning metrizability of pointwise paracompact Moore spaces, Canad. J. Math., 16 (1964), 407-411.

64. H. H. Wicke and J. M. Worrell Jr., Characterizations of developable spaces, Canad. J. Math., 17 (1965), 820-830.

Received March 7, 1974.

DUKe UNIVERSITY 



\section{PACIFIC JOURNAL OF MATHEMATICS}

\section{EDITORS}

RICHARD ARENS (Managing Editor)

University of California

Los Angeles, California 90024

\section{R. A. Beaumont \\ University of Washington \\ Seattle, Washington 98105}

\section{J. DugundJI}

Department of Mathematics

University of Southern California

Los Angeles, California 90007

D. Gilbarg and J. Milgram

Stanford University

Stanford, California 94305

\section{ASSOCIATE EDITORS}
E. F. BECKENBACH
B. H. NeumanN
F. WOLF
K. YOSHIDA

\section{SUPPORTING INSTITUTIONS}

UNIVERSITY OF BRITISH COLUMBIA
CALIFORNIA INSTITUTE OF TECHNOLOGY
UNIVERSITY OF CALIFORNIA
MONTANA STATE UNIVERSITY
UNIVERSITY OF NEVADA
NEW MEXICO STATE UNIVERSITY
OREGON STATE UNIVERSITY
UNIVERSITY OF OREGON
OSAKA UNIVERSITY

UNIVERSITY OF BRITISH COLUMBIA

UNIVERSITY OF CALIFORNIA

MONTANA STATE UNIVERSITY

NEW MEXICO STATE UNIVERSITY

OREGON STATE UNIVERSITY

OSAKA UNIVERSITY

\author{
UNIVERSITY OF SOUTHERN CALIFORNIA \\ STANFORD UNIVERSITY \\ UNIVERSITY OF TOKYO \\ UNIVERSITY OF UTAH \\ WASHINGTON STATE UNIVERSITY \\ UNIVERSITY OF WASHINGTON \\ AMERICAN MATHEMATICAL SOCIETY \\ NAVAL WEAPONS CENTER
}

The Supporting Institutions listed above contribute to the cost of publication of this Journal, but they are not owners or publishers and have no responsibility for its content or policies.

Mathematical papers intended for publication in the Pacific Journal of Mathematics should be in typed form or offset-reproduced, (not dittoed), double spaced with large margins. Underline Greek letters in red, German in green, and script in blue. The first paragraph or two must be capable of being used separately as a synopsis of the entire paper. Items of the bibliography should not be cited there unless absolutely necessary, in which case they must be identified by author and Journal, rather than by item number. Manuscripts, in triplicate, may be sent to any one of the editors. Please classify according to the scheme of Math. Reviews, Index to Vol. 39. All other communications should be addressed to the managing editor, or Elaine Barth, University of California, Los Angeles, California, 90024.

The Pacific Journal of Mathematics expects the author's institution to pay page charges, and reserves the right to delay publication for nonpayment of charges in case of financial emergency.

100 reprints are provided free for each article, only if page charges have been substantially paid. Additional copies may be obtained at cost in multiples of 50 .

The Pacific Journal of Mathematics is issued monthly as of January 1966. Regular subscription rate: $\$ 72.00$ a year (6 Vols., 12 issues). Special rate: $\$ 36.00$ a year to individual members of supporting institutions.

Subscriptions, orders for back numbers, and changes of address should be sent to Pacific Journal of Mathematics, 103 Highland Boulevard, Berkeley, California, 94708.

PUBLISHED BY PACIFIC JOURNAL OF MATHEMATICS, A NON-PROFIT CORPORATION

Printed at Kokusai Bunken Insatsusha (International Academic Printing Co., Ltd.), 270, 3-chome Totsuka-cho, Shinjuku-ku, Tokyo 160, Japan.

Copyright (C) 1973 by Pacific Journal of Mathematics Manufactured and first issued in Japan 


\section{Pacific Journal of Mathematics \\ Vol. 55, No. $2 \quad$ October, 1974}

Walter Allegretto, On the equivalence of two types of oscillation for elliptic

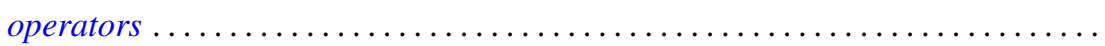

Edward Arthur Bertram, A density theorem on the number of conjugacy classes in

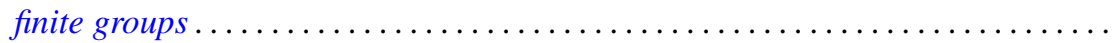

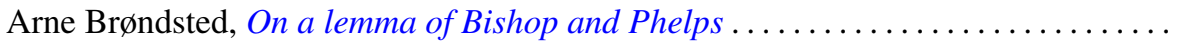

Jacob Burbea, Total positivity and reproducing kernels ..................

Ed Dubinsky, Linear Pincherle sequences . . . . . . . . . . . . . . . . . .

Benny Dan Evans, Cyclic amalgamations of residually finite groups .............

361

Barry J. Gardner and Patrick Noble Stewart, A "going down" theorem for certain

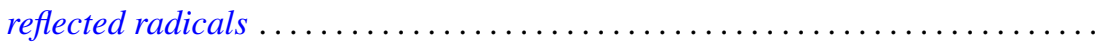

381

Jonathan Light Gross and Thomas William Tucker, Quotients of complete graphs:

revisiting the Heawood map-coloring problem ....................

Sav Roman Harasymiv, Groups of matrices acting on distribution spaces .........

Robert Winship Heath and David John Lutzer, Dugundji extension theorems for

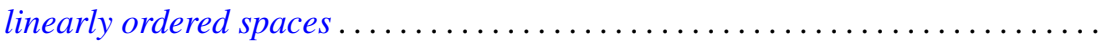

Chung-Wu Ho, Deforming p. l. homeomorphisms on a convex polygonal

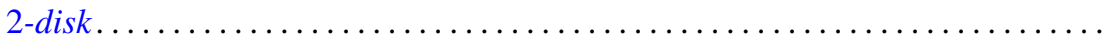

Richard Earl Hodel, Metrizability of topological spaces .................

Wilfried Imrich and Mark E. Watkins, On graphical regular representations of

cyclic extensions of groups .......................... 461

Jozef Krasinkiewicz, Remark on mappings not raising dimension of curves ..... . 479

Melven Robert Krom, Infinite games and special Baire space extensions . . . . . . 483

S. Leela, Stability of measure differential equations . . . . . . . . . . . . . . . . 489

M. H. Lim, Linear transformations on symmetric spaces . . . . . . . . . . . . . . . 499

Teng-Sun Liu, Arnoud C. M. van Rooij and Ju-Kwei Wang, On some group algebra modules related to Wiener's algebra $M_{1} \ldots \ldots \ldots \ldots \ldots \ldots \ldots \ldots \ldots \ldots \ldots$

Dale Wayne Myers, The back-and-forth isomorphism construction ............ 521

Donovan Harold Van Osdol, Extensions of sheaves of commutative algebras by

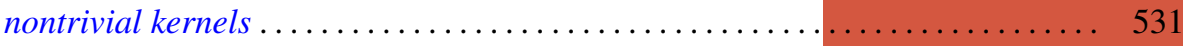

Alan Rahilly, Generalized Hall planes of even order ................... 543

Joylyn Newberry Reed, On completeness and semicompleteness of first countable

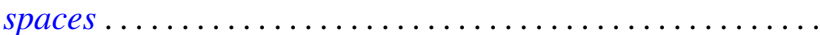

Alan Schwartz, Generalized convolutions and positive definite functions associated

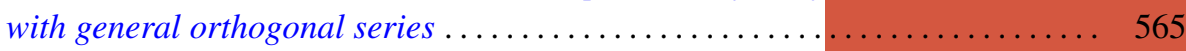

Thomas Jerome Scott, Monotonic permutations of chains . . . . . . . . . . . 583

Eivind Stensholt, An application of Steinberg's construction of twisted groups .... 595

Yasuji Takeuchi, On strongly radicial extensions . . . . ................. 619

William P. Ziemer, Some remarks on harmonic measure in space . . . . . . . . . . 629

John Grant, Corrections to: “Automorphisms definable by formulas” . . . . . . . . 639

Peter Michael Rosenthal, Corrections to: "On an inversion for the general

Mehler-Fock transform pair" ......................... 640

Carl Clifton Faith, Corrections to: "When are proper cyclics injective” . . . . . . 640 